AIP Applied Physics

\title{
Ultrafast optical modification of magnetic anisotropy and stimulated precession in an epitaxial Co2MnAl thin film
}

Y. Liu, L. R. Shelford, V. V. Kruglyak, R. J. Hicken, Y. Sakuraba et al.

Citation: J. Appl. Phys. 101, 09C106 (2007); doi: 10.1063/1.2711702

View online: http://dx.doi.org/10.1063/1.2711702

View Table of Contents: http://jap.aip.org/resource/1/JAPIAU/v101/i9

Published by the American Institute of Physics.

\section{Related Articles}

Mechanism of reversing the Neel domain walls in the Co nanostripes with transverse magnetic anisotropy Appl. Phys. Lett. 101, 252412 (2012)

Structural and magnetic properties of biaxially textured NiFe2O4 thin films grown on c-plane sapphire J. Appl. Phys. 112, 123910 (2012)

Thick CoFeB with perpendicular magnetic anisotropy in $\mathrm{CoFeB}-\mathrm{MgO}$ based magnetic tunnel junction AIP Advances 2, 042182 (2012)

Ferromagnetic enhancement and magnetic anisotropy in nonpolar-oriented ( $\mathrm{Mn}, \mathrm{Na})$-codoped $\mathrm{ZnO}$ thin films Appl. Phys. Lett. 101, 242401 (2012)

The nature of $\mathrm{Cr}$ center in $\mathrm{GaN}$ : Magnetic anisotropy of $\mathrm{GaN}: \mathrm{Cr}$ single crystals

J. Appl. Phys. 112, 113914 (2012)

\section{Additional information on J. Appl. Phys.}

Journal Homepage: http://jap.aip.org/

Journal Information: http://jap.aip.org/about/about_the_journal

Top downloads: http://jap.aip.org/features/most_downloaded

Information for Authors: http://jap.aip.org/authors

\section{ADVERTISEMENT}

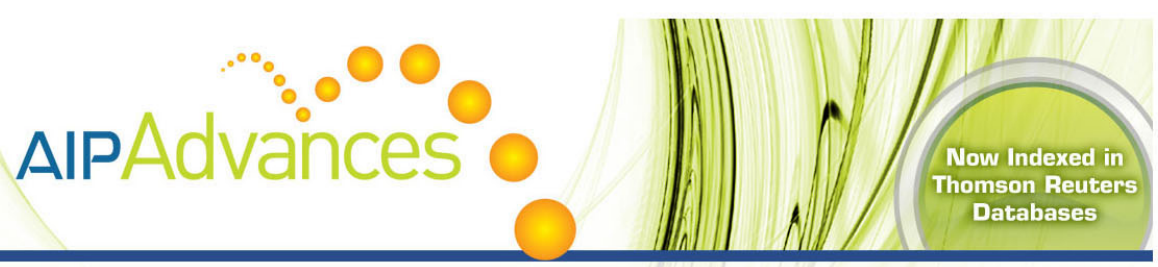

\section{Explore AIP's open access journal: Rapid publication \\ - Article-level metrics \\ Post-publication rating and commenting}




\title{
Ultrafast optical modification of magnetic anisotropy and stimulated precession in an epitaxial $\mathrm{Co}_{2} \mathrm{MnAl}$ thin film
}

\author{
Y. Liu, ${ }^{\text {a) }}$ L. R. Shelford, V. V. Kruglyak, and R. J. Hicken \\ School of Physics, University of Exeter, Stocker Road, Exeter EX4 4QL, United Kingdom \\ Y. Sakuraba, M. Oogane, Y. Ando, and T. Miyazaki \\ Department of Applied Physics, Graduate School of Engineering, Tohoku University, Sendai 980-8579, \\ Japan
}

(Presented on 11 January 2007; received 1 November 2006; accepted 13 December 2006; published online 2 May 2007)

\begin{abstract}
An all-optical pump-probe method was used to study magnetization precession in an epitaxial $\mathrm{Co}_{2} \mathrm{MnAl}$ Heusler alloy thin film. The frequency and amplitude of precession showed a clear fourfold variation as the orientation of the static field was applied in different directions within the plane of the film, revealing that the precession is induced by an ultrafast modification of the magnetocrystalline anisotropy field. The effective fields acting upon the magnetization have been determined and the damping parameter is found to decrease rapidly as the strength of the applied field is increased. (C) 2007 American Institute of Physics. [DOI: 10.1063/1.2711702]
\end{abstract}

Heusler alloys show great promise for use in spintronic devices due to their predicted half-metallic band structure. Recently, large room temperature tunnel magnetoresistance (TMR) values have been observed in magnetic tunnel junctions (MTJs) with $\mathrm{Co}_{2} \mathrm{MnAl}$ (Ref. 1) and other Heusler alloy ${ }^{2,3}$ electrodes. It is expected that the half-metallic band structure may suppress spin-flip processes and hence reduce the damping of the magnetization precession. Ferromagnetic resonance (FMR) has previously been used to study the intrinsic Gilbert damping parameter of thin $\mathrm{Co}_{2} \mathrm{MnAl}$ films. ${ }^{4,5}$ However, time domain measurements of the damping parameter over a continuous range of frequencies are needed to achieve a comprehensive understanding of the dynamic properties while measurements within the low field regime are of particular technological relevance. All-optical pumpprobe techniques have been demonstrated in the last decade that enable ultrafast demagnetization, coherent magnetization rotation, and hot electron relaxation in magnetic thin films ${ }^{6-11}$ to be studied over an extended range of frequencies.

In this paper, we present all-optical pump-probe measurements of precessional magnetization dynamics in an epitaxial $\mathrm{Co}_{2} \mathrm{MnAl}$ thin film. By varying the strength and orientation of the static magnetic field and fitting the resulting transient Kerr rotation signals to a macrospin solution of the Landau-Lifshitz-Gilbert (LLG) equation, we have studied the variation of the frequency, amplitude, phase, and damping of the precession. We demonstrate that precession is induced by an ultrafast modification of the magnetocrystalline anisotropy and determine the dependence of the damping upon both the strength and orientation of the magnetic field.

$\mathrm{A} \mathrm{MgO}(001) / \mathrm{Cr}(40 \mathrm{~nm}) / \mathrm{Co}_{2} \mathrm{MnAl}(30 \mathrm{~nm}) / \mathrm{MgO}(10 \mathrm{~nm})$ structure was grown by magnetron sputtering at room temperature on a $\mathrm{MgO}(001)$ substrate. ${ }^{12}$ The sample was postannealed at $300{ }^{\circ} \mathrm{C}$ so that epitaxial $\mathrm{Co}_{2} \mathrm{MnAl}$ (001) was obtained with the $B 2$ lattice structure. ${ }^{1,12}$ Optical pump-probe measurements were made upon the samples with $130 \mathrm{fs}$

a)Electronic mail: yanwei.liu@exeter.ac.uk pulses of $800 \mathrm{~nm}$ wavelength from a Ti:sapphire regenerative amplifier. The $p$-polarized pump pulse had energy of up to $0.8 \mu \mathrm{J}$ and was directed onto the sample surface at near normal incidence. The response of the sample magnetization was determined from a time resolved magneto-optical Kerr effect (TRMOKE) rotation measurement made with an $s$-polarized $400 \mathrm{~nm}$ wavelength probe pulse, with energy of $4 \mathrm{~nJ}$, incident at an angle of $40^{\circ}$. The pump and probe beams were focused onto the sample surface with spot sizes of 140 and $80 \mu \mathrm{m}$, respectively, and overlapped as they were viewed with a high-magnification charge-coupled device (CCD) camera.

Following excitation by the pump beam, the magnetization and magnetic anisotropy of the film undergo an ultrafast modification. As a result, the effective magnetic field acting upon the magnetization may no longer be parallel to the magnetization. The resulting torque stimulates precession of the magnetization which may be described by the LLG equation,

$$
\frac{\partial \mathbf{M}}{\partial t}=-|\gamma| \mathbf{M} \times \mathbf{H}_{\mathrm{eff}}+\frac{\alpha}{M}\left(\mathbf{M} \times \frac{\partial \mathbf{M}}{\partial t}\right),
$$

where $\alpha$ is the Gilbert damping parameter, $\gamma=2.80 \times \pi$ $\times 10^{6} \times g \mathrm{~Hz} / \mathrm{Oe}$ is the gyromagnetic ratio of the electron, and $g$ is the spectroscopic splitting factor. The effective magnetic field $\mathbf{H}_{\text {eff }}$ includes the applied static magnetic field, the demagnetizing field, and contributions due to the magnetocrystalline anisotropy. For the case of a small amplitude uniform precession, an algebraic expression for the time dependent magnetization may be obtained if the magnetocrystalline anisotropy is assumed to be instantaneously reduced by the optical pumping. When the static field is applied parallel to the plane of incidence of the probe, the Kerr rotation signal contains an oscillatory component due to the out-of-plane component of the dynamic magnetization. Alternatively, if the static field is applied perpendicular to the plane of incidence, the Kerr signal results from the sum of two oscillatory terms that are linearly proportional to the 
components of magnetization that lie within the plane of incidence. ${ }^{13}$ In either case the oscillatory Kerr rotation has the form

$$
\phi_{K} \propto \Delta \phi \cos \left(2 \pi f_{0} t+\varphi\right) \exp (-t / \tau),
$$

where $\Delta \phi, f_{0}, \tau$, and $\varphi$ are the amplitude, frequency, decay constant, and initial phase of the signal, respectively. The value of $\varphi$ is dependent on the experimental geometry, while in the limit $\alpha \ll 1, \Delta \phi, f_{0}$, and $\tau$ can be expressed algebraically as

$$
\begin{aligned}
& \Delta \phi=\frac{-(1 / 2) \sin \left[4\left(\phi-\phi_{4}\right)\right] \Delta\left(K_{1} / M_{s}\right)}{H \cos (\phi)+\left(K_{1} / 2 M_{s}\right) \cos \left[4\left(\phi-\phi_{4}\right)\right]}, \\
& f_{0}=\frac{1}{2 \pi}|\gamma|\left(H_{\alpha} H_{\beta}\right)^{1 / 2}, \\
& \tau=\frac{2}{|\gamma|\left(H_{\alpha}+H_{\beta}\right) \alpha}, \\
& H_{\alpha}=H \cos (\phi)+\frac{K_{1}}{2 M_{s}}\left\{3+\cos \left[4\left(\phi-\phi_{4}\right)\right]\right\}+4 \pi M_{s}, \\
& H_{\beta}=H \cos (\phi)+\frac{2 K_{1}}{M_{s}} \cos \left[4\left(\phi-\phi_{4}\right)\right] .
\end{aligned}
$$

Here $\phi$ and $\phi_{4}$ are the angles that the magnetization and [100] axis of the $\mathrm{Co}_{2} \mathrm{MnAl}$ describe with the applied static field H. $M_{S}$ and $K_{1}$ are the saturation magnetization and cubic magnetocrystalline anisotropy constant, respectively, while the value of $\phi$ is determined from the static equilibrium condition,

$$
M H \sin (\phi)+\frac{K_{1}}{2} \sin \left[4\left(\phi-\phi_{4}\right)\right]=0 .
$$

Measurements were first performed with the magnetic field $\left(\mathbf{H}_{L}\right)$ applied in the plane of the sample and parallel to the plane of incidence, as shown in Fig. 1(a). A typical timeresolved rotation Kerr signal is shown in Fig. 1(b), for which the magnetic field of $852 \mathrm{Oe}$ was applied at $20^{\circ}$ relative to the [110] axis of the $\mathrm{Co}_{2} \mathrm{MnAl}$. The signal consists of an oscillatory component superimposed upon a component due to an ultrafast demagnetization. By comparing fine scans of the initial rise of the signal with the longitudinal hysteresis loop (not shown), the peak demagnetization was found to be $5 \%$. It has been demonstrated that the amplitude and phase of the magnetization precession may be strongly dependent on the helicity of the pump beam for garnet films studied in the Faraday geometry. ${ }^{14}$ However, the amplitude and phase of the demagnetization and precessional components were found to be independent of the helicity of the pump in our investigation, while the precession amplitude was linearly dependent on the pulse energy, as shown in the inset of Fig. 1(b).

In order to eliminate the contribution from the demagnetization signal, the experiments were mainly conducted with the static field $\left(\mathbf{H}_{T}\right)$ applied perpendicular to the plane of incidence, as shown in Fig. 1(a). Figure 2(a) shows the transient Kerr rotation signals obtained in this transverse field (a)
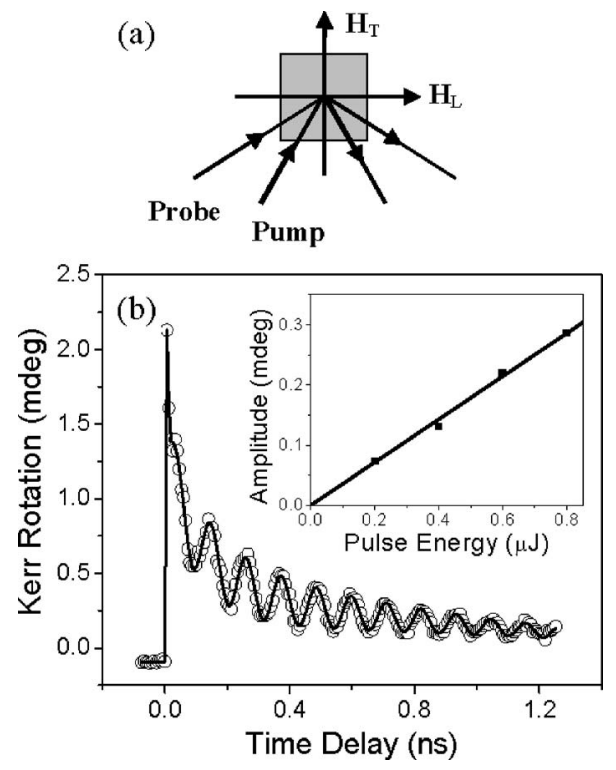

FIG. 1. (a) Schematic diagram of the experimental configuration with a longitudinal $\left(\mathbf{H}_{L}\right)$ or a transverse $\left(\mathbf{H}_{T}\right)$ magnetic field. (b) Typical time resolved Kerr rotation signal obtained with $\mathbf{H}$ parallel to the plane of incidence. The magnetic field of 852 Oe was applied at an angle of $20^{\circ}$ relative to the [110] axis of the $\mathrm{Co}_{2} \mathrm{MnAl}$. The inset shows the dependence of the precession amplitude on the pulse energy, where the solid line is a guide to the eye.

geometry as a static field of $211 \mathrm{Oe}$, sufficient to saturate the sample, was applied in different directions relative to the [110] axis of the $\mathrm{Co}_{2} \mathrm{MnAl}$. The transient Kerr rotation signals show that the precession amplitude depends strongly on the orientation of the static magnetic field. The raw timeresolved signals were fitted to the sum of a damped sinusoid [Eq. (2)] and to a small exponentially decaying background that accounts for the slow recovery of the magnetic anisotropy. The saturation magnetization was set to the bulk value of $730 \mathrm{emu} / \mathrm{cm}^{3}$.,12 Equations (3), (4), and (6)-(8) were used to fit the amplitude and frequency of the precession of the magnetization, shown in Figs. 2(b) and 2(c), respectively. Figure 2(b) shows that the precession amplitude has a clear fourfold variation with the orientation of the static field. No
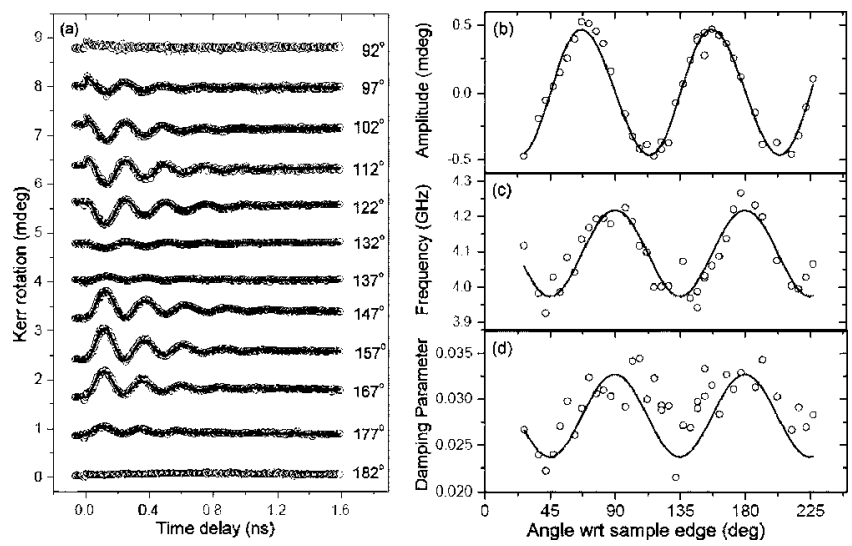

FIG. 2. (a) Transient Kerr rotation signals obtained with a magnetic field of $211 \mathrm{Oe}$ applied at difference angles to the [110] axis of the $\mathrm{Co}_{2} \mathrm{MnAl}$ and perpendicular to the plane of incidence. (b) The dependence of the precession amplitude, (c) the precession frequency, and (d) the damping parameter on the orientation of the magnetic field are shown. 

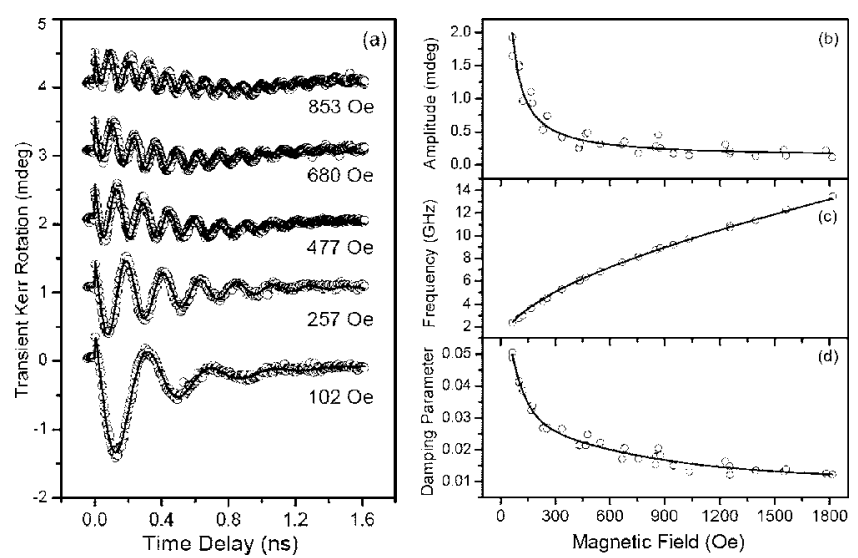

FIG. 3. (a) Transient Kerr rotation signals with $\mathbf{H}$ perpendicular to the plane of incidence and at $20^{\circ}$ to the $\mathrm{Co}_{2} \mathrm{MnAl}[110]$ axis. (b) The dependence of the precession amplitude, (c) the precession frequency, and (d) the damping parameter on the strength of the magnetic field (the solid line is a guide to the eye, while (b) and (c) show fits to the macrospin model).

precession was observed with the field applied parallel to (easy axis) or $45^{\circ}$ from (hard axis) the [110] direction, while the maximum amplitude was found for the field applied midway between these orientations. The precession signal is also seen to undergo a $180^{\circ}$ phase change as the static field crosses the easy or hard axis. From Fig. 2(c), the precession frequency is seen to exhibit a fourfold variation which has been fitted to Eq. (4). The fourfold variation of the precession amplitude and frequency with the orientation of the magnetic field confirms that the magnetization precession is induced by an ultrafast optical modification of the magnetocrystalline anisotropy, ${ }^{9,15}$ leading to a reorientation of the effective field acting upon the magnetization. When the static field is applied along either the easy or hard axis, no precession is induced because the effective field remains parallel to the magnetization after excitation. In all other orientations $\mathbf{H}_{\text {eff }}$ rotates suddenly from alignment parallel to $\mathbf{M}$, producing a torque on $\mathbf{M}$. The damping parameter $\alpha$ calculated using Eq. (5) shows a weak fourfold variation to which a sine curve has been added as a guide to the eye. However, we note that the extracted values of $\alpha$ are slightly modified if the frequency of the fitted function is allowed to vary with time so as to account for the slow recovery of the magnetocrystalline anisotropy, and so further measurements are required to determine whether the observed fourfold variation is an intrinsic property of the sample or an artifact of the fitting procedure.

Figure 3(a) shows the dependence of the transient Kerr rotation signal upon the strength of the magnetic field. Here the static field was applied at $20^{\circ}$ relative to the $\mathrm{Co}_{2} \mathrm{MnAl}$ [110] easy axis. The transient precession signals were again fitted using Eq. (2), and the fitted parameter values are summarized in Figs. 3(b)-3(d). As the magnetic field is increased, the precession frequency increases while the precession amplitude and the damping parameter $\alpha$ decrease. The solid lines in Figs. 3(b) and 3(c) are fits to Eqs. (3) and (4), respectively. By fitting both the field and angle dependence data simultaneously, best fit values of $g=2.10$ and $K_{1}=$ $-4572 \mathrm{erg} / \mathrm{cm}^{3}$ were determined. A value of $\alpha$ $=0.015 \pm 0.003$ is obtained at a frequency of $10 \mathrm{GHz}$, which is twice the value obtained in FMR measurements at the same frequency. ${ }^{4}$ A number of authors have reported a field dependent damping parameter and suggested a variety of underlying mechanisms. ${ }^{16-20}$ The damping parameter extracted from the fits reported here should be regarded as a phenomenological parameter that accounts for the combined effect of intrinsic damping, inhomogeneous broadening, two magnon scattering, any higher order spin waves processes, and propagation effects resulting from the nonuniform spatial profile of the precession. Further work is now required to understand which of these mechanisms make a significant contribution to the damping observed in the present study.

We have used an all-optical pump-probe method to study the magnetization dynamics of a thin $\mathrm{Co}_{2} \mathrm{MnAl}$ Heusler alloy film. The frequency and amplitude of the observed precessional oscillations showed a clear fourfold variation, revealing that the magnetization precession is induced by an ultrafast modification of the magnetocrystalline anisotropy field. By fitting the Kerr signal to a macrospin model, we have determined the values of the effective fields acting upon the magnetization and shown that the value of the damping parameter decreases rapidly as the strength of the applied field is increased.

The authors would like to acknowledge the support of the New Energy and Industrial Technology Development Organization (NEDO) and the Engineering and Physical Sciences Research Council (EPSRC).

${ }^{1}$ Y. Sakuraba, J. Nakata, M. Oogane, Y. Ando, H. Kato, A. Sakuma, T. Miyazaki, and H. Kubota, Appl. Phys. Lett. 88, 022503 (2006).

${ }^{2}$ S. Kämmerer, A. Thomas, A. Hütten, and G. Reiss, Appl. Phys. Lett. 85, 79 (2004).

${ }^{3}$ Y. Sakuraba, M. Hattori, M. Oogane, Y. Ando, H. Kato, A. Sakuma, T. Miyazaki, and H. Kubota, Appl. Phys. Lett. 88, 192508 (2006).

${ }^{4}$ R. Yilgin, M. Oogane, S. Yakata, Y. Ando, and T. Miyazaki, IEEE Trans. Magn. 41, 2799 (2005).

${ }^{5}$ M. Oogane, T. Wakitani, S. Yakata, R. Yilgin, Y. Ando, A. Sakuma, and T. Miyazaki, Jpn. J. Appl. Phys., Part 1 45, 3889 (2006).

${ }^{6}$ E. Beaurepaire, J. C. Merle, A. Daunois, and J. Y. Bigot, Phys. Rev. Lett. 76, 4250 (1996).

${ }^{7}$ J. Hohlfeld, E. Matthias, R. Knorren, and K. H. Bennemann, Phys. Rev. Lett. 78, 4861 (1997).

${ }^{8}$ M. van Kampen, C. Jozsa, J. T. Kohlhepp, P. LeClair, L. Lagae, W. J. M. de Jonge, and B. Koopmans, Phys. Rev. Lett. 88, 227201 (2002).

${ }^{9}$ Q. Zhang, A. V. Nurmikko, A. Anguelouch, G. Xiao, and A. Gupta, Phys. Rev. Lett. 89, 177402 (2002).

${ }^{10}$ A. Kimel, A. Kirilyuk, A. Tsvetkov, R. V. Pisarev, and T. Rasing, Nature (London) 429, 850 (2004).

${ }^{11}$ V. V. Kruglyak, R. J. Hicken, M. Ali, B. J. Hickey, A. T. G. Pym, and B. K. Tanner, Phys. Rev. B 71, 233104 (2005).

${ }^{12}$ H. Kubota, J. Nakata, M. Oogane, Y. Ando, H. Kato, A. Sakuma, and T. Miyazaki, J. Appl. Phys. 97, 10C913 (2005).

${ }^{13}$ J. Wu, J. R. Moore, and R. J. Hicken, J. Magn. Magn. Mater. 222, 189 (2000).

${ }^{14}$ F. Hansteen, A. Kimel, A. Kirilyuk, and T. Rasing, Phys. Rev. B 73, 014421 (2006).

${ }^{15}$ H. B. Zhao et al., Appl. Phys. Lett. 86, 152512 (2005).

${ }^{16}$ W. Platow, A. N. Anisimov, G. L. Dunifer, M. Farle, and K. Baberschke, Phys. Rev. B 58, 5611 (1998).

${ }^{17}$ J. P. Nibarger, R. Lopusnik, and T. J. Silva, Appl. Phys. Lett. 82, 2112 (2003).

${ }^{18}$ M. L. Schneider, T. Gerrits, A. B. Kos, and T. J. Silva, Appl. Phys. Lett. 87, 072509 (2005).

${ }^{19}$ M. Djordjevic, G. Eilers, A. Parge, M. Münzenberg, and J. S. Moodera, J. Appl. Phys. 99, 08F308 (2006).

${ }^{20}$ J. Wu, N. D. Hughes, J. R. Moore, and R. J. Hicken, J. Magn. Magn. Mater. 241, 96 (2002). 